\title{
Observation of Equilibria with a Double Magnetic Axis in LHD
}

\author{
H.Yamada ${ }^{1,2}$, Y.Suzuki ${ }^{1}$, K.Ida ${ }^{1,2}$, M.Yoshinuma ${ }^{1}$, T.Kobuchi ${ }^{1}$, \\ K.Y.Watanabe ${ }^{1,2}$, K. Tanaka $^{1}$ and LHD Experimental Group \\ ${ }^{1}$ National Institute for Fusion Science, Toki, Gifu 509-5292, Japan \\ ${ }^{2}$ Graduate University for Advanced Studies, SOKENDAI, \\ Hayama, Kanagawa 240-0193, Japan
}

\section{Introduction}

Plasma shape control is a major knob to investigate confinement as well as MHD characteristics of magnetically confined plasmas. In particular, plasma elongation has been widely explored in many experiments and theoretical investigations. For example, optimization in the line of W7-AS and W7-X owes much to reduction of Pfrisch-Schlueter currents due to elongation [1]. It is also widely recognized that plasma elongation affects energy confinement in tokamaks, which can be seen in the ITER H-mode scaling [2].

On the other hand, the role of separatrix has been attracting interest in both a fundamental issue of a dynamical system with cross of stable and unstable manifolds and applied issues for performance of magnetic confinement. Many reports can be referred to the latter issues, which are related to dynamics of magnetic islands [3] and its effect on transport [4], equilibrium $\beta$ limit and a function of divertor.

This article reports the observation of equilibrium with an internal separatrix in the confinement domain, which is realized by an extreme elongation in the Large Helical Device (LHD) [5]. LHD has a large flexibility to explore a configuration effect by using 6 independent coil systems and elongation can be controlled by a quadrupole field. Throughout this study, elongation is characterized by the toroidal averaged ellipticity $\kappa$ of the last closed magnetic surface and $\kappa$ of larger than 1 and smaller than 1 means that plasma is elongated vertically and horizontally in the toroidal average, respectively. Standard configuration in LHD has the averaged ellipticity $\kappa$ of 1 while it should be note that the elliptic last closed flux surface of local $\kappa$ of approximately 2 rotates poloidally.

A study on effect moderate elongation $(0.8<\kappa<1.4)$ has shown that confinement is degraded gradually in both prolate (vertically elongated) and oblate (horizontally elongated) configurations [6]. Since the rotational transform is weaker in the core region than in the periphery in LHD, modulation by poloidal field is more effective inside. Therefore plasma elongation is more pronounced towards the magnetic axis. A theoretical analysis suggests that excess quadrupole field results in split of the magnetic axis. Then in the prolate case the internal separatrix emerges and produces figure-8 petal structure with a double magnetic axis. In the oblate case, lying figure- 8 structure is not clear and a double magnetic axis is degenerated. Figure 1 shows the vacuum magnetic surfaces in a wide range of elongation.

Although analytics based on stellarator expansion is available for this complex geometry [7], it does not provide real physics related to internal separatrix sufficiently due its crude assumptions. Finite- $\beta$ effect is also of much importance. A computation with assumption of existence of concentric nested flux surfaces such as VMEC [8] cannot deal with these 
complex equilibria, either. However, the 3-D MHD equilibrium code HINT [9] does not need any assumption of flux surfaces, therefore, it can reproduce a realistic equilibrium which can be directly compared with experimental observations. Calculation by HINT indicates that MHD equilibrium with a double magnetic axis does exist in LHD. Finite- $\beta$ effect on these configurations has been studied [10].

In this study, first the finite- $\beta$ equilibrium with a double magnetic axis is demonstrated both experimentally and numerically. Then finite- $\beta$ effect on the petal structure and confinement performance in the outer domain of the separatrix is discussed in the prolate case, and also characteristics of an enhanced Shafranov shift and consequent equilibrium $\beta$ limit are discussed in the oblate case.
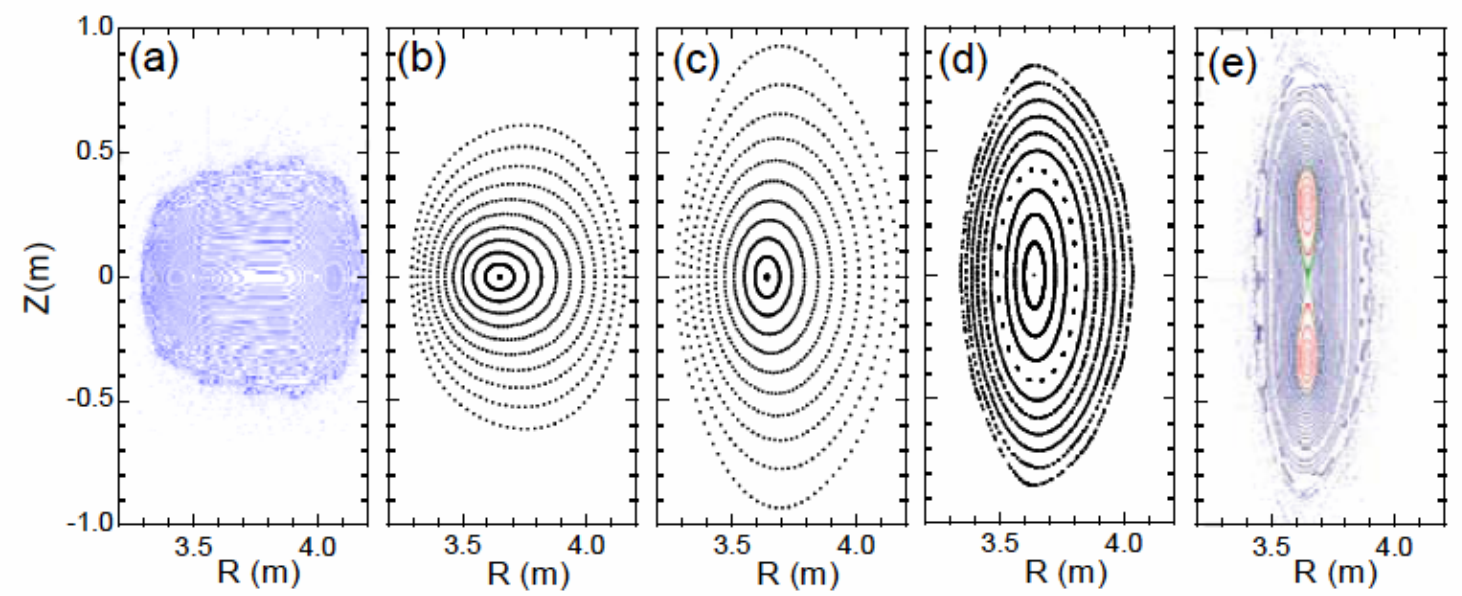

Fig.1 Change of vacuum magnetic surfaces with elongation (a) $\kappa=0.5$, (b) $\kappa=0.8$, (c) $\kappa=1.0$, (d) $\kappa=1.4$, and (e) $\kappa=2.0$

\section{Comparison of analytical prediction and HINT in the prolate case}

Figure 2 illustrates the characterization of evolution of internal separatrix in the prolate case. Magnetic flux surface can be elongated by quadrupole field and further application of quadrupole field eventually realizes a doublet configuration with a double magnetic axis bounded by the separatrix. The height of the petal from the equatorial plane where the $\mathrm{X}$ point of separatrix is located is defined by $H_{s}$ here. When $H_{s}$ is not zero, a double magnetic axis is formed. When $H_{s}$ reaches $a$, boundary of the petal with a figure-8 shape becomes the last closed flux surface.

An analytics based on Ref.7 suggests that critical elongation to split the magnetic axis is given by

$\kappa_{c}^{4}=1+\frac{4 t_{0}}{t_{a}-t_{0}}$,

where $t_{0}$ and $t_{a}$ are the rotational transform at the magnetic axis and the last closed flux surface, respectively. As shown in Fig.3 (a), this simple analytics suggests that split of the magnetic axis starts at the ellipticity $\kappa$ of 1.3 and the separatrix bounded configuration is formed at $\kappa$ of 1.6 .

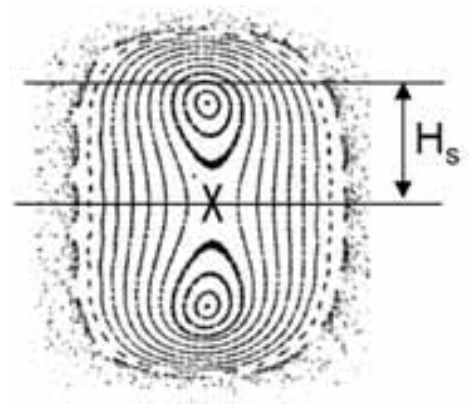

Fig.2 Definition of petal height. 
Figure 3(a) also shows the results from the HINT with zero $\beta$. The critical point to produce the split of the magnetic axis agrees with the analytics while the evolution of the petal is much mitigated compared with the analytics. The separatrix bounded plasma is formed beyond 4 .

The analytics also predicts that magnetic shear is reversed inside the petal bounded by separatrix. While rotational transform at the separatrix is stagnated to zero, that at magnetic axis increases with elongation, which means that tokamak like rotational transform is generated in the petal. Figure 3(b) shows the evolution of the rotational transform at the axis with the increase of $\kappa$ calculated by the analytics and HINT. The trends themselves agree to each other while the rate of the increase is much mitigated in the calculation by HINT again. Together with the evolution of $H_{s}$, the analytics based on stellarator expansion provides reasonable picture qualitatively, however it overestimates the effect of elongation since HINT should incorporate physics of MHD equilibrium in its formalism much better than a simple physics.

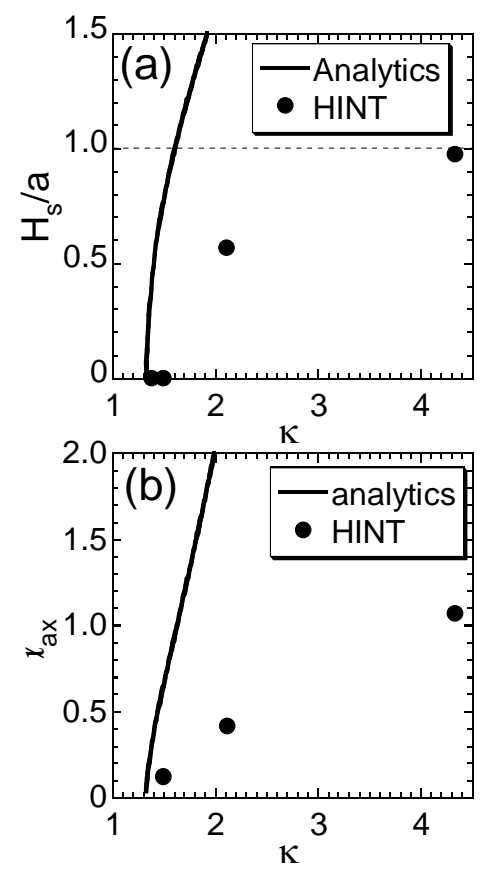

Fig.3 Evolution of (a) petal height and (b) rotational transform as a function of $\kappa$.

\section{Experimental observation and its comparison with HINT}

NBI heated plasmas with large elongation in both vertical (prolate) and horizontal (oblate) directions have been investigated in LHD. Figure 4 shows the 2-D pictures by a tangential X-ray camera [11]. In addition to clear shaping of the outline, figure 8 structure can be seen clearly in the strongly prolate case $(\kappa=2.0)$. This is due to the fact that the $\mathrm{X}$ point is stagnated on the equatorial plane. On the other hand, in the oblate case $(\kappa=0.5)$, the existence of X point, in other words, lying figure 8 structure is not clear since the petal structure has a radial excursion along the torus and its image becomes dim by the path integral effect along the tangential chord of the camera.
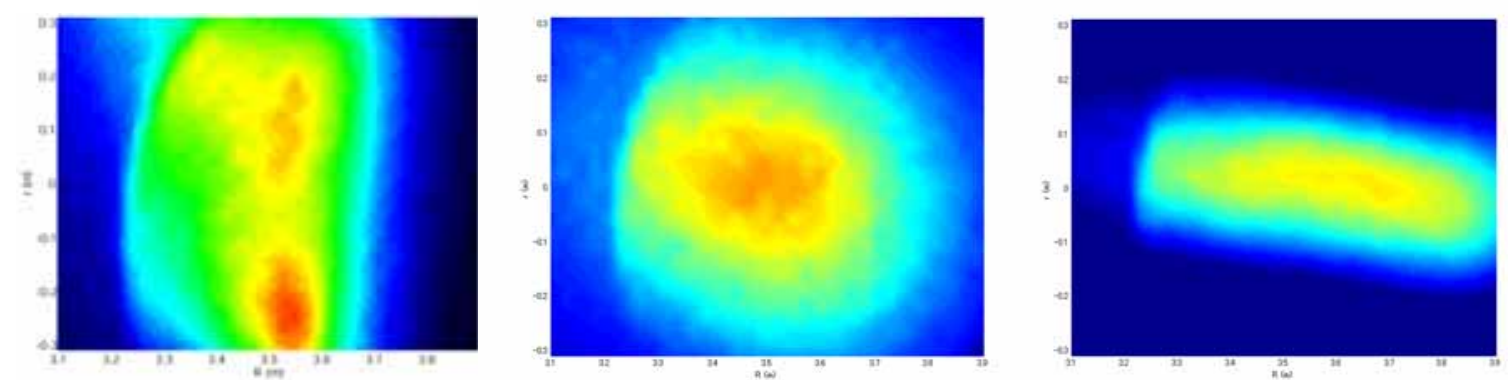

Fig.4 Tangential images by a soft X-ray camera of NBI heated plasmas in three configurations. The elongation $\mathrm{k}$ are 2.0, 1.0 and 0.5 from the right to the left.

\section{(1) Prolate configuration}

Although the 2-dimensional profile of temperature and density are not available at 
present, breakdown of assumption of concentric surfaces with a single magnetic axis is observed in the line averaged density measurement with different chord geometry. One measurement chord passes vertically through two petals and magnetic axes and another chord passes horizontally through the X-point with avoiding the petal. When the concentric magnetic surfaces are formed, these two measurements coincide with each other with correction of only path length. Dependence of the ratio of these two measurement on the stored energy in the case of $\kappa=2.0$ is shown in Fig.5(a), which suggests that deviation from the simple concentric geometry is enhanced by the stored energy.

Finite- $\beta$ equilibrium calculation by HINT has indicated that the up and down separation of the petal is enhanced with $\beta$ and simultaneously erogodization outside the petal proceeds [10]. Consequently, only the petal region preserves confinement capability. This trend reflects difference in the line averaged pressures along the vertical chords passing the petals and horizontal chord avoiding the petals. Figure 5 (b) shows the evolution of the ratio of those line averaged pressure with $\beta$. The discrepancy of these two values is enhanced with $\beta$ and this trend is consistent with
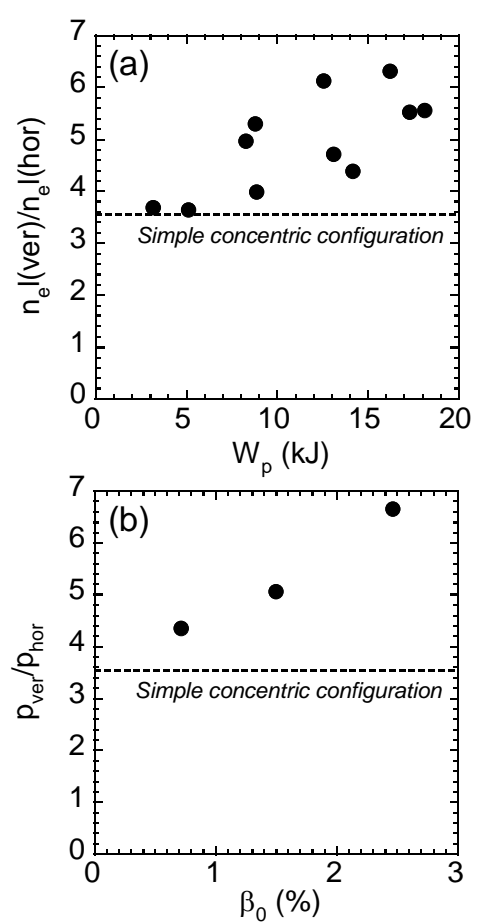

Fig.5 Ratio of physical quantities along vertical and horizontal chords. (a) line averaged density in NBI heated plasma with $\kappa$ of 2.0 , (b) line averaged pressure in the corresponding calculation by HINT.

experimental observation although the compared physical quantity is not exactly the same, i.e., density in the experiment and pressure in the computation.

\section{(2) Oblate configuration}

Since the Shafranov shift is enhanced by horizontal elongation [12], equilibrium $\beta$ limit is lowered in oblate configuration. In general, the equilibrium $\beta$ limit is defined by the Shafranov shift as large as a half the minor radius, when the deformation of magnetic flux surface is thought not to be tolerable. However, this is not physically reasonable definition. In the tokamak case, the separatrix is generated inboard side due to enhanced Pfrish-Schlueter currents [15]. Oblate configuration provides the experimental evidence what happens by a large Shafranov shift and can mitigate the approach to the question what the equilibrium $\beta$ limit is.

Figure 6 shows the electron temperature profile in the experiment and the pressure profile calculated by HINT in the case of $\kappa$ of 0.5 . Both profiles are plotted along the major radius. As shown in Fig.6 (a), the Shafranov shift beyond a half the minor radius is already observed in low $\beta(0.3 \%)$. Along with the increase in $\beta$, inflection of temperature gradient appears inboard side of the peak. This evolution of profile is well reconstructed by the HINT calculation. The calculation of HINT indicates that large Shafranov shift occurs even in low $\beta$ since the separatrix geometry already exists in the vacuum condition and the rotational 
transform is close to zero. With increase in $\beta$, the outer petal evolves while the inner petal degenerates. The outer region of the petal, namely the outside of the internal separatrix becomes stochastic and loses confinement capability. This trend is pronounced in the inboard side of the outer petal in the physical space. While large pressure gradient is maintained in the outer petal (outer separatrix domain), the pressure gradient declines in the outside of this region. This physical picture seems to be consistent with the experimental observation. The reason why the experimental observation in the inboard side is somewhat mitigated from the prediction by HINT may be healing of ergodization due to unresolved dynamics of the plasma [13] or tolerable parallel heat transport.

Although the direct measurement of the change of magnetic geometry is not available, circumstantial evidence supports the validity of the calculation by HINT. Subsequently the picture of equilibrium $\beta$ limit can described based on results from the calculation by HINT. Figure 7 (a) shows the Shafranov shift as a function of $\beta$. In the case of regular configuration $(\kappa=1)$, the Shafranov shift increases proportionally with $\beta$ (see the dotted curve) in the available operational range. In the case of large horizontal elongation $(\kappa=0.5)$, the drastic change can be seen and this change may be categorized in three phases. In the phase $I$, the Shafranov shift increases with $\beta$ typically below a half the minor radius. In the phase II, the outer petal plays an essential role of confinement region. Since the Shafranov shift in the petal is reduced due to enhanced rotational transform with reversed shear. Then the peaking of pressure profile in the petal due to erogodization enhances the Shafranov shift (phase III). These characteristics are closely linked with the loss of confinement volume. Figure 7 (b) shows declination of the confinement volume inside the good flux surface as a function of the Shafranov shift. In phase I, the volume decreases gradually by ergodization in the periphery. In phase II, the

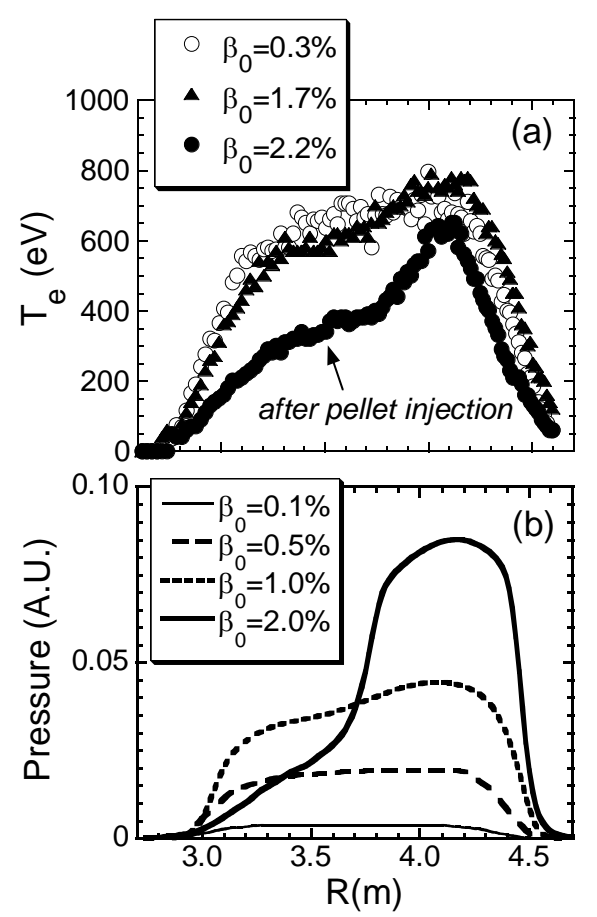

Fig.6 Profile evolution in the configuration with $\kappa$ of 0.5 . (a) Electron temperature in NBI heated plasmas measured by Thomson scattering, (b) Pressure profile calculated by HINT.
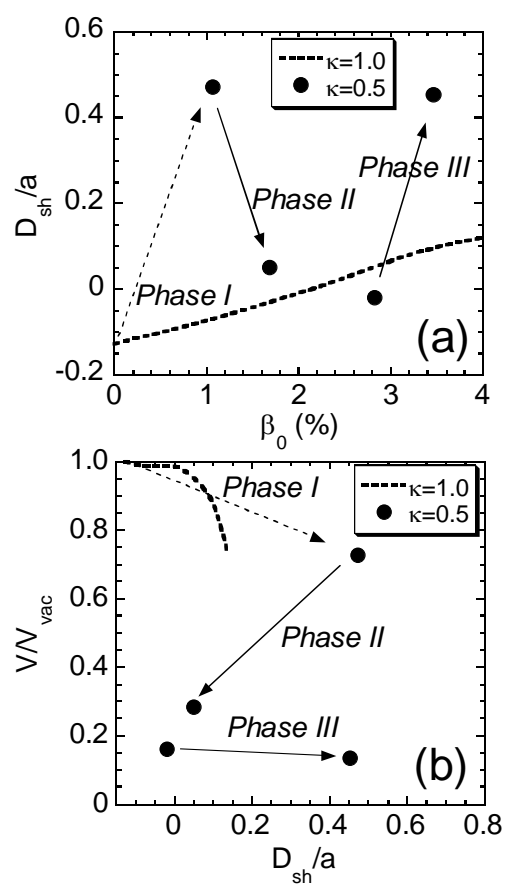

Fig.7 Change of equilibrium calculated by HINT. (a) Shafranov shift as a function of the central $\beta$, (b) Volume of good magnetic surface as a function of Shafranov shift. 
ergodization proceeds quickly due to evolution of the internal separatrix domain. Large volume is lost despite of the small Shafranov shift in the petal. Then the confinement volume decreases gradually again with the Shafranov shift in the petal (phase III).

\section{Summary}

Exploration of eccentric magnetic configurations with a double axis has started in LHD. Finite- $\beta$ equilibrium with internal separatrix has been demonstrated in both NBI heated plasma experiment and computation by HINT which does not need assumption of existence of nested flux surfaces. Experimental observations such as tangential images, electron density measurements by horizontal and vertical chords (prolate case), and electron temperature measurements along the major radius (oblate case) agree well with prediction from HINT. The picture of equilibrium $\beta$ limit when Shafranov shift exceeds a half the radius has been proposed.

\section{Acknowledgements}

This work is supported by NIFS05ULHH515 from the budget grant-in-aid of the National Institute for Fusion Science.

\section{References}

[1] J.Geiger et al., Fusion Sci. Technol. 46 (2004) 13.

[2] ITER PHYSICS BASIS, Nucl. Fusion 39 (1999) 2175.

[3] N.Ohyabu et al., Phys. Rev. Lett. 88 (2002) 055005.

[4] S.Inagaki et al., Phys. Rev. Lett. 92 (2004) 055002

[5] A.Iiyoshi et al., Nucl. Fusion 39 (1999) 1659.

[6] H.Yamada et al., Plasma Physics and Control. Fusion 43 (2001) A55.

[7] V.D.Pustovitov, Review of Plasma Physics (2000, Kluwer Academic/Plenum Publisher) Vol.21, pp.138.

[8] S.P.Hirshman Comput. Phys. Commun. 43 (1986) 143.

[9] K.Harafuji et al., J. Comput. Phys. 81 (1989) 169

[10] Y.Suzuki et al., in Fusion Energy 2004 (Proc. 20th Int. Conf. Vilamoura, 2004) (Vienna: IAEA) CD-ROM file TH/P2-31 and http://www-naweb.iaea.org/napc/physics/fec/fec2004/datasets/index.html

[11] Y.Liang et al., Rev. Sci. Instrum. 72 (2001) 717.

[12] T.Kobuchi et al., in Proc. 31st EPS Conference on Plasma Phys. London, 28 June - 2 July 2004 ECA Vol.28G, P-5.115 (2004)

[13] K.Narihara et al., Phys. Rev. Lett. 87 (2001) 135002 